\title{
Instrument Hacking: Repurposing and Recoding a Multi-well Instrument for Automated, High-throughput Monochromatic UV Photooxidation of Organic Compounds
}

Sean A. MacIsaac ${ }^{1}$, Crystal L. Sweeney ${ }^{1}$, and Graham A. Gagnon ${ }^{1}$

${ }^{1}$ Centre for Water Resources Studies, Department of Civil and Resource Engineering, Dalhousie University, Halifax, NS, Canada

${ }^{*}$ Corresponding Author: Graham A. Gagnon

Email: graham.gagnon@dal.ca

Address: Department of Civil and Resource Engineering, Dalhousie University, 1360 Barrington St. Halifax, NS, Canada (B3H 4R2)

Phone: +1 9024946070

Date: 20 September 2020 


\section{Supplementary Material}

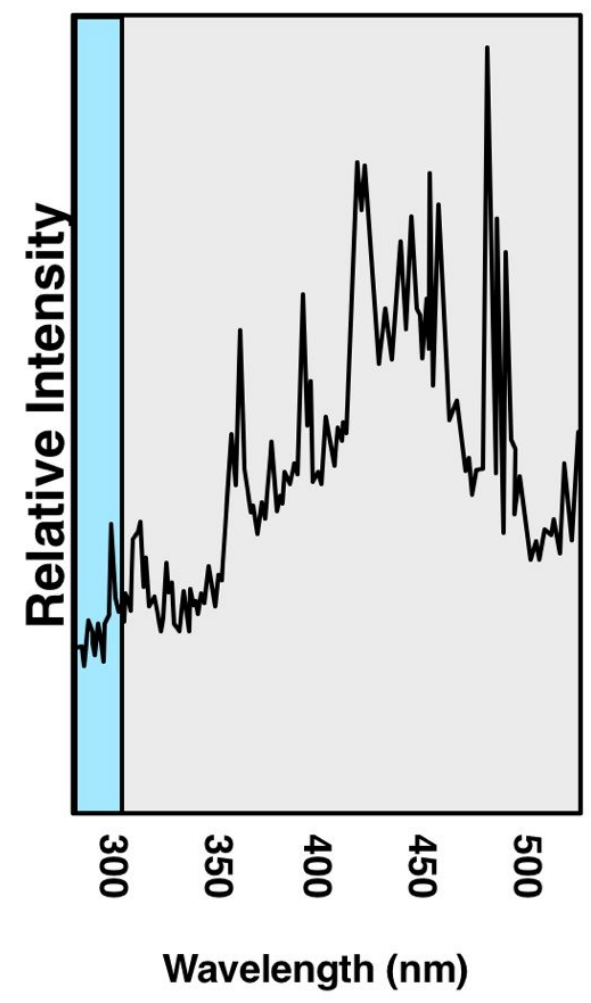

Figure 1: Relative output of the microplate reader xenon-flash light source with the UV region designated in blue ${ }^{11}$

Equation S1. Moles of iron for exposed actinometry samples

$$
\text { Moles of } \mathrm{Fe}^{2+}=\frac{\left[A_{510}(\text { Sample })-A_{510}(\text { Blank })\right]}{11,110}
$$

Equation S2. Einsteins of UV light absorbed at a given wavelength

$$
\text { einsteins of UV absorbed at wavelength } \lambda=\frac{\text { moles of } F e^{2+} \text { generated }}{\phi_{\text {known }}}
$$


Equation S3. Joules per einstein at a given wavelength

$$
U_{\lambda}\left(\text { einstein }^{-1}\right)=\frac{h c N_{A}}{\lambda}
$$

Equation S4. UV power output at a given wavelength

$$
P(W)=\frac{U_{\lambda}\left(\text { einstein }^{-1}\right) \times \text { einsteins of } U V \text { absorbed at wavelength } \lambda}{(1-R) \times(t(s))}
$$

Equation S5. UV fluence calculation for a given wavelength

$$
\text { UV Fluence }\left(\mathrm{mJ} \mathrm{cm}^{-2}\right)=\left[\frac{P(W) \times 1000\left({ }^{\mathrm{mW}} / W\right) \times t(s)}{A\left(\mathrm{~cm}^{2}\right)}\right] \times \text { Ref factor }_{\text {f }} \times \text { Area }_{\text {factor }}
$$

Table 1: UV fluence regression equations for $255 \mathrm{~nm}$ to $280 \mathrm{~nm}$ where $t$ is in seconds of UV exposure.

\begin{tabular}{lll}
\hline Wavelength (nm) & $\begin{array}{l}\text { Fluence Equation } \\
\left(\mathbf{m J \bullet c ^ { - 2 }}\right)\end{array}$ & $\mathbf{R}^{\mathbf{2}}$ \\
\hline $\mathbf{2 5 5}$ & $0.42 t+44.93$ & .929 \\
\hline $\mathbf{2 6 0}$ & $0.85 t+56.78$ & .797 \\
\hline $\mathbf{2 6 5}$ & $1.39 t+35.85$ & .955 \\
\hline $\mathbf{2 8 0}$ & $1.56 t+17.06$ & .849 \\
\hline
\end{tabular}

